\title{
Digestive Toxicities Secondary to Immune Checkpoint Inhibition Therapy - Reports of Rare Events. A Systematic Review
}

\author{
Liliana Radulescu ${ }^{1,2}$, Dana Crisan ${ }^{1,2}$, Cristiana Grapa ${ }^{1}$, Dan Radulescu ${ }^{1,2}$
}

1) Iuliu Hatieganu University

of Medicine and Pharmacy,

Faculty of Medicine, Internal

Medicine Department, Cluj-

Napoca;

2) Clinical Municipal Hospital,

Cluj-Napoca, Romania

\begin{abstract}
Background \& Aims: While immune checkpoint inhibitors therapy (ICI) is exceedingly effective, these drugs are associated with various immune-related adverse effects. As gastrointestinal, hepatic or pancreatic toxicity becomes more common, various reports of rare adverse effects have emerged, leading to a significant clinical and prognostic impact. We aimed to provide a systematic review of mainly case-reports on rare events, to help physicians to make an accurate and fast diagnosis.

Methods: We performed a systematic review of the literature, using established MeSH terms: "immune checkpoint inhibitors", "gastrointestinal tract", "gastrointestinal diseases", "liver", "pancreas", "nivolumab", "ipilimumab", the subheadings "adverse effects", "toxicity" and the supplementary concepts "pembrolizumab", "tremelimumab", "atezolizumab", "avelumab", "durvalumab”, with defined inclusion criteria.

Results: From 419 manuscripts initially selected, 74 reports of rare adverse events were included in our review. Special cases of neutrophilic gastritis, hemorrhagic gastritis, or even perforations were described at upper digestive tract. Different types of colitis were found secondary to ICI such as pseudomembranous, granulomatous, collagenous and microscopic colitis or even inflammatory bowel disease. In terms of liver toxicity, we found rare reports of cholangitis, granulomatous hepatitis, lipodystrophy and hepatic sinusoidal obstruction syndrome. Pancreas toxicity was rarely reported as severe pancreatitis, exocrine failure and diabetes mellitus.

Conclusion: Although a complete check-up of every organ at every routine visit may not be practical, focus on symptoms, targeted laboratory and imaging testing may reveal rare organ damage. Raising awareness of the uncommon toxicities related to the immunotherapy is essential, as some rare events can lead to fatal outcomes.
\end{abstract}

Key words: immune checkpoint inhibitors - gastrointestinal tract - adverse effects - side effects - liver pancreas.

Abbreviations: CMV: cytomegalovirus; CTLA-4: cytotoxic T-lymphocyte associated protein 4; IBD: inflammatory bowel disease; ICI: immune checkpoint inhibitor; MRCP: magnetic resonance cholangiopancreatography; PD-1: programmed cell death-1; PDL-1: programmed cell death ligand protein 1.

\section{INTRODUCTION}

Received: 17.04 .2021 Accepted: 08.10.2021
The hypothesis that the immune system could aid in the combat against cancer has been in existence since the $19^{\text {th }}$ century [1] and since then, the work of scientists led to the approval of the first immune checkpoint inhibitor (ICI) for the treatment of melanoma in 2011 [2]. In the tumor microenvironment, the checkpoint inhibitors block the antitumor $\mathrm{T}$ cell mediated defense mechanism, thus inhibiting the development of the malignant tumor. Two main immune checkpoint molecules that block anti-tumor immunity are known: one of them is cytotoxic T-lymphocyte associated protein 4 (CTLA-4), the other is programmed cell death-1 (PD-1) and related to it programmed cell death ligand protein 1 (PDL-1). Recently, researchers have identified antibodies that were able to inhibit these molecules, being useful in treating different types of cancers. The anti-CTLA- 4 antibodies approved and used are ipilimumab and tremelimumab, the anti-PD-1 inhibitors are represented by nivolumab and pembrolizumab, and the PD-L1 inhibitors are atezolizumab, avelumab and durvalumab $[3,4]$. These monoclonal antibodies, named ICIs, are efficient in treating melanoma, non-small cell lung cancer, lymphomas and renal cancer. Compared to the classical antineoplastic agents, ICIs are more efficient 
in treating neoplasia, by blocking the tumor growth and prolonging the survival. Immune checkpoint inhibitors are human or humanized IgG globulins. First ICI approved was ipilimumab for metastatic melanoma treatment; further, it was approved for metastatic colon cancer, renal cell carcinoma, hepatocellular carcinoma and non-small cell lung cancer. The first PD-1 inhibitor approved is represented by nivolumab, for the treatment of metastatic melanoma and non-small cell lung cancer, but also with proved efficacy in Hodgkin lymphoma, hepatocellular carcinoma and metastatic renal carcinoma. Pembrolizumab, another PD-1 inhibitor, is approved for the treatment of melanoma, metastatic non-small cell lung cancer, metastatic head, and neck squamous carcinoma. Recently, PDL1 ICIs, like atezolizumab, durvalumab and avelumab have been approved in metastatic urothelial carcinomas [5-10]. Indications approved by the Food and Drug Administration for ICIs treatment are summarized in Fig. 1 [11].

Immune checkpoint inhibitors toxicity can have multiple side effects, namely dermatological, gastrointestinal, endocrine, respiratory, cardiac, renal, ophthalmological, and neurological. The mechanism of their toxicity is not completely understood, but studies revealed differences between anti CTLA-4 and anti-PD1 either in severity or location. For instance, colitis is more severe in patients treated with anti CTLA-4, and in general, the toxicities related to anti CTLA-4 are more severe [12]. A study suggested the potential role of tertiary lymphoid structures as predictors for immunotherapy, meaning that the presence of tumor associated CD20 B cells together with CD8 $\mathrm{T}$ cells improved survival [13]. As the digestive toxicity is one of the most prevalent, special attention needs to be paid to the manifestations that are also rarely reported, but are more than fearful, as they can end-up in a life-threatening event [14]. The most important digestive toxicities are hepatic and gastro- intestinal, but the pancreas can also be involved. It is worth mentioning that the prevalence of digestive toxicities is higher in patients treated with anti-CTLA-4 (27-54\%) [15] while in anti-PD-1 is $19.2 \%$. When using a combined therapy, the toxicity reaches $44.1 \%$ [16]. Manifestation of gastrointestinal side effects can happen at any time during treatment, but it is more frequent in the first 6 to 8 weeks of initiation [17].

The main difference between conventional chemotherapy and immunotherapy regarding the side effects is that while chemotherapy leads to immunosuppression and cytotoxicity, most adverse reactions related to ICI treatment are due to immune activation [18]. Once toxicity occurs, special treatment should be given, namely immunosuppressants and ICI therapy should be discontinued for a while until reaching the symptoms resolution, or should be stopped completely, depending on the severity of the side effects. We provide a systematic review on primarily case reports and case series of unusual or rare case reports on toxicity associated with ICI treatment. Our aim is to increase awareness of all the possible events that may occur during cancer treatment, since some of them can even be fatal. As the use of ICIs is significantly intensifying, physicians must be aware of all possible side effects, including the rare ones, for a proper diagnosis and management.

\section{METHODS}

Our study was completed following the Preferred Reporting Items for Systematic Reviews and Meta-Analysis (PRISMA) criteria. We performed a systematic literature search of the PubMed database, using the MeSH terms: "immune checkpoint inhibitors", "gastrointestinal tract", "gastrointestinal diseases", "liver", "pancreas", "nivolumab", “ipilimumab”, the subheadings "adverse effects", "toxicity" and the supplementary

\section{U.S. FDA APPROVED IMMUNE-CHECKPOINT INHIBITORS}

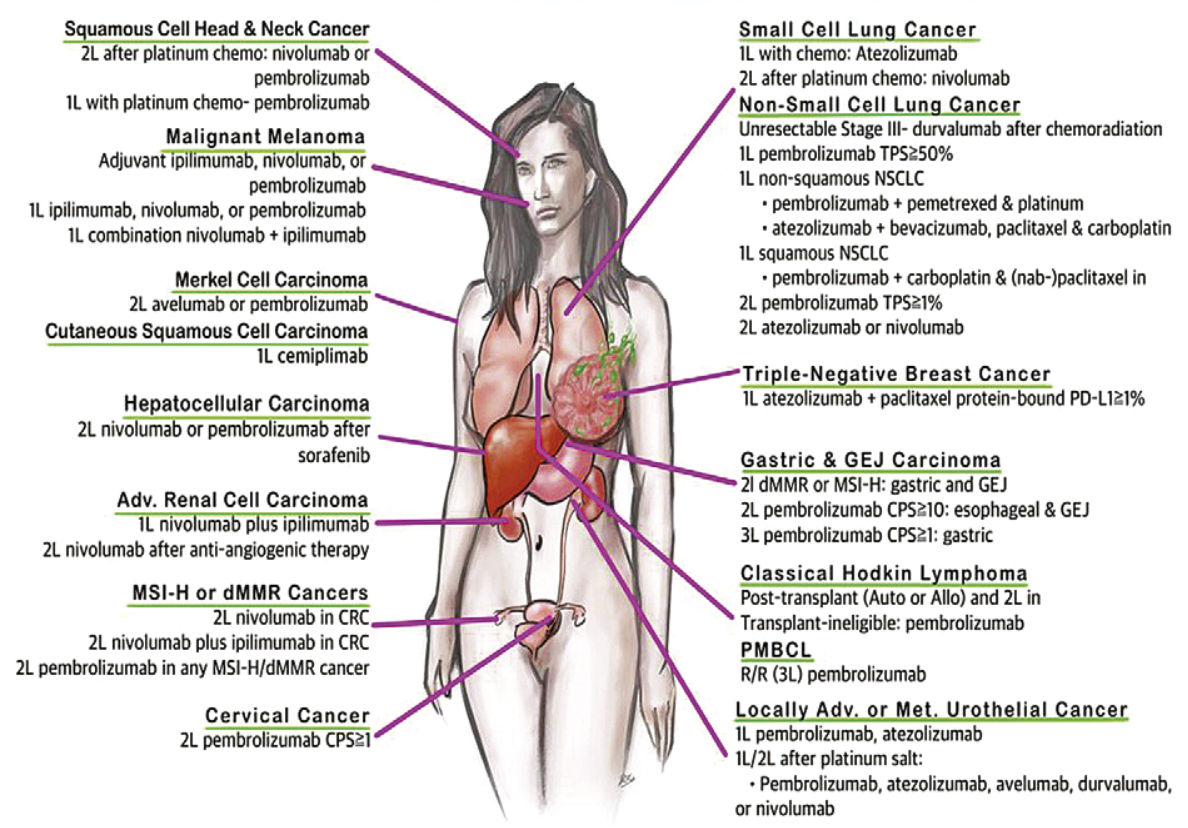

Fig. 1. Food and Drug Administration approved indications for checkpoint inhibitor therapy. Reprinted with permission from [11]. 
concepts "pembrolizumab", "tremelimumab”, "atezolizumab", "avelumab", "durvalumab", in different combinations. The date of the last search was 10 July 2021.

Our inclusion criteria were represented by 1) English written studies; 2 ) case-reports or case series of cancer patients treated with ICI; 3 ) a causal role between the administration of ICI and the adverse effect was established; 4) the cases included details about the type of cancer; type of ICI used; investigations and diagnosis procedure; management of the adverse effect; outcome of the patients; 5 ) the cases revealed unusual or rare reports. Other types of studies besides case reports were also included if they met the other criteria for inclusion. Three separate reviewers assessed the studies for eligibility criteria. All articles that did not meet the inclusion criteria listed above were excluded. Since epidemiology studies on prevalence and incidence of the rare side effects of ICI therapy on the gastrointestinal tract, liver or pancreas are scarce, our review inclusion process was based on the peer-review system of the cases included that established them as "rare", "unusual".

\section{RESULTS}

The selection process is detailed in Fig. 2. Initially 419 studies were identified. After removing the duplicates, 379 studies remained for further analysis. A number of 186 studies were excluded based on title and their abstract, leaving 190 to be assessed for eligibility by full text reading. After assessment, 74 studies which comprised of predominantly case-reports, remained eligible for inclusion. We found various rare casereports regarding the gastrointestinal tract, the liver and the pancreas, with different outcomes from well-responders to corticosteroids to fatal events; the reports are summarized in Supplementary file.

\section{Gastrointestinal Tract Toxicity}

We found five cases of ulcerative esophagitis after nivolumab, pembrolizumab, ipilimumab or durvalumab therapy for different types of cancer [19-23], with patients presenting different outcomes from well-responders to corticosteroids to fatal events. Unusual cases of gastritis were reported, such as steroid refractory [24] or neutrophilic gastritis [25] under nivolumab therapy. Lymphocytic gastritis (acute form at five years after introduction of ICI [26]), [27] and cytomegalovirus (CMV) gastritis [28] induced by pembrolizumab were reported. More severe cases such as hemorrhagic gastritis [29] in a patient treated with nivolumab or two cases of asymptomatic gastric perforation, one of them leading to a life-threating event were also described [30, 31]. Celiac disease has been reported as a rare event; incidence of $0.05 \%$ after ICI treatment, most of cases with good symptom resolution after dietary changes [32]. Another peculiar case, of late onset asymptomatic gastro-duodenitis associated
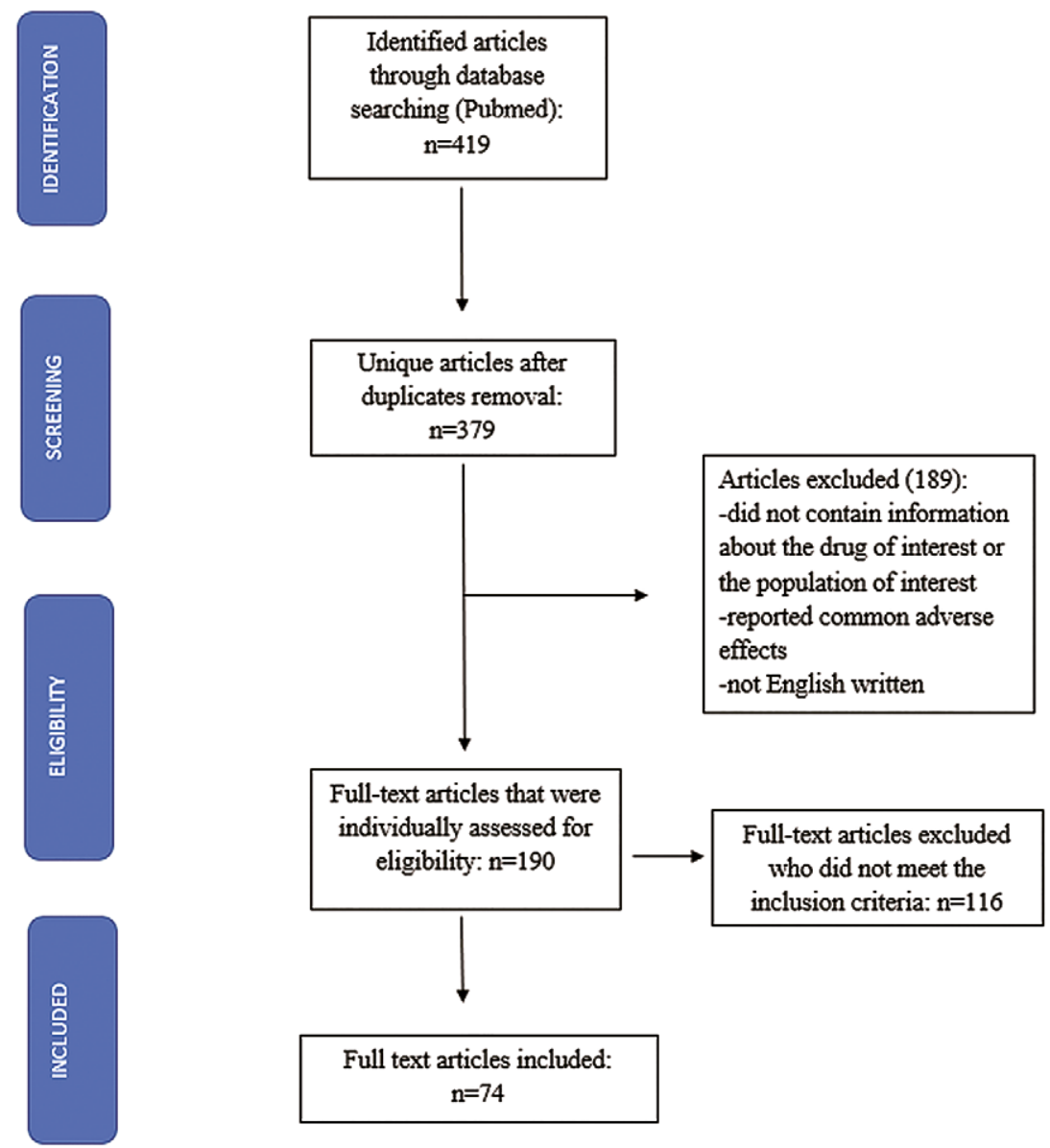

Fig. 2. PRISMA flow diagram for the systematic review 
with cholangitis, with good response to corticosteroids was described in a melanoma patient following nivolumab therapy [33].

Lower gastrointestinal tract rare adverse effects are welldocumented. Our search led us to various cases of severe refractory enterocolitis that responded only to infliximab therapy [34, 35] and a case of infliximab refractory colitis. While cases of severe colitis associated with anti CTLA-3 therapy are rather frequent, severe cases after anti PD-1 therapy are scarcely reported, with an incidence of only $0.4 \%$ [36]. Some unusual cases of colitis were also described, such as collagenous [37, 38], pseudomembranous [39], granulomatous [40], microscopic colitis [41] or even inflammatory bowel diseases (IBD) [42-44] due to different types of ICI.

A case of asymptomatic appendicitis after dual therapy (nivolumab and ipilimumab) therapy [45] diagnosed through a routine positron emission tomography (PET) pointed out the specter of immune related events that can be missed or only diagnosed by chance; another interesting case described a small bowel perforation due to fast tumor regression secondary to nivolumab and ipilimumab treatment [46]. Other four cases of patients under nivolumab, ipilimumab or atezolizumab therapy developed small bowel perforation, a rare, but potentially fatal event [47-50]. A single case reported the appearance of eosinophilic enteritis in a melanoma patient under dual therapy [51]; other types of enteritis that were rarely reported: protein losing enteropathy [52], widespread enteropathy [53], apoptotic enteropathy $[54,55]$. Two cases of intestinal pseudo-obstruction in patients treated with nivolumab or pembrolizumab added to the diversity of side effects that can occur $[56,57]$. Two cases of myenteric plexus neuropathy after dual therapy or single nivolumab treatment were reported, one of the cases led to sepsis and death in a 66-year-old female treated for Merkle cell carcinoma [58, 59].

\section{Liver toxicity}

Liver injury due to ICI treatment is usually described as a hepatitis pattern, but ICI induced cholangitis was also reported, as a rare side effect $(0.05 \%$ prevalence) $[60]$. A fatal case of sclerosing cholangitis caused by nivolumab was described in a 77-year-old male who did not respond to corticosteroids [60]. A single case of non-alcoholic fatty liver disease and lipodystrophy also related to nivolumab treatment was described [61]. A histopathological study on the liver of a pembrolizumab treated patient revealed liver injury with predominantly biliary damage [62], and another clinicopathological study showed patterns of granulomatous hepatitis, sclerosing cholangitis or lymphocytic duct injury, adding to the spectrum of ICI induced liver injury, besides hepatitis [63]. Additionally, other uncommon side effects emerged, with only two case reports published, of hepatic sinusoidal obstruction syndrome related to nivolumab treatment $[64,65]$, and a case of vanishing bile duct syndrome potentially related to pembrolizumab [66].

\section{Pancreatic toxicity}

Although pancreatic enzymes are commonly elevated in ICI treated patients, pancreatitis is a rare event. It has also been documented that PD-1 and PD-L1 inhibitors precipitate diabetes in animal models; a review found a $0.9 \%$ prevalence of treatment induced insulin-dependent diabetes [67]. We found various reports of PD-1/PD-L1 induced diabetes mellitus [68-74]. A peculiar presentation of pembrolizumab induced pancreatitis was described as a small bowel perforation [75]. Few cases of ICI induced exocrine pancreatic insufficiency pointed out that this diagnosis must be kept in mind in patients presenting with diarrhea, since not all cases are caused by colitis. A team of researchers also correlated pancreatic atrophy with exocrine pancreatic insufficiency and diarrhea in this category of patients [76].

\section{DISCUSSION}

Main upper gastrointestinal symptoms revealing toxicity in ICI treated patients are anorexia, nausea, vomiting, abdominal pain, abdominal bloating. Upper gastrointestinal symptoms may be secondary to toxic immune damage of the stomach, duodenum, or small bowel. Involvement of these organs may occur within 1 or 2 months after starting the therapy, or later in the course of therapy, after months or even years (the latest ICI induced side effect our search found was after 5 years of therapy [26]). Endoscopy may be of help in documenting upper gastrointestinal involvement (inflammation, ulcers and perforations in more severe cases). Treatment of gastrointestinal symptoms consists of discontinuing immunotherapy until symptom resolution, or continuing it, depending on the case, and initiating corticosteroid therapy. Additionally, anti TNF-alfa drugs, such as infliximab can be helpful in treating gastrointestinal toxicity for patients who do not respond to corticosteroids $[78,79]$. Upper gastrointestinal adverse effects are less common and poorly described, and it appears that they are frequently related to PD-1 inhibitors, in contrast to lower gastrointestinal side effects, which are reported in a third of patients treated with CTLA-4 inhibitors [80, 81].

Five case reports described ulcerative esophagitis as a sideeffect after nivolumab, combined treatment with nivolumab and ipilimumab, pembrolizumab or durvalumab therapy. The cases had very different outcomes, two were rapidly improved by corticosteroids $[19,20]$, one developed an esophageal stenosis and responded to tocilizumab treatment [21] and, unfortunately, a patient treated with pembrolizumab died [22]. Another patient treated with pembrolizumab who developed esophagitis had complete resolution of symptomatology after anti-PD-1 therapy cessation [23]. These outcomes reveal the importance of diagnosis and prompt treatment of any possible side-effect, as the same diagnosis can lead to different outcomes, including life threating complications.

There are various cases of gastritis reported in literature after ICI treatment, and while most of them responded to corticosteroids, there were some of them refractory to this therapy. A 16-year-old melanoma patient treated with nivolumab developed steroid-refractory gastritis that responded only to infliximab therapy [24]. Pembrolizumab has been found to induce autoimmune gastritis in a melanoma patient, five years after start of therapy [26] contributing to the multitude of late-onset ICI related events. Pembrolizumab was also incriminated in two unusual cases of lymphocytic gastritis and CMV gastritis $[27,28]$, revealing a larger spectrum of 
histological findings in gastritis patients. The peculiarity of CMV gastritis case is as well represented by an opportunistic infection appearing in an immune-competent patient and the need for ganciclovir therapy for ICI induced gastritis. The authors presenting this case explained the paradox through the immune reconstitution inflammatory syndrome that occurs in patients with HIV infection or tuberculosis. It seems that the PD/PD-L1 pathway impedes proper functioning of CD4 and CD8 T cells, thus permitting the virus to persist. When blocking this pathway, there could be a boost in virus specific $\mathrm{T}$ cell activity and consequently activate a latent infection.

A single case of neutrophilic gastritis associated to nivolumab treatment was also described [25]. Another case report of a 77-year-old male treated with nivolumab for metastatic lung cancer raised awareness on an additional rare type of gastritis associated with ICI treatment: hemorrhagic gastritis [29]. In this case, treatment with prednisolone led to the symptoms' improvement. As the use of nivolumab becomes more frequent, vigilance for such side effects is required.

A peculiar consequence was described in a 43-year-old patient diagnosed with cutaneous melanoma treated with nivolumab, i.e the development of late onset severe gastroduodenitis diagnosed by PET before any symptom would have appeared (confirmed endoscopically as a grade 3 gastroduodenitis) and ten days later, she additionally developed a grade 4 cholestasis that proved to be cholangitis after evaluation through magnetic resonance cholangiopancreatography (MRCP) and liver biopsy. The gastric symptoms responded well to corticosteroids, but for cholangitis, an immune-suppression intensification with mycophenolate mofetil or anti-TNF was considered. Still, the treatment was not initiated because of a systemic infection with Staphylococcus aureus [33].

The causal relation between ICI treatment and celiac disease occurrence was not certainly proved $[82,83]$. A cohort study encompassing 377 melanoma patients undergoing ICI treatment reported a $0.05 \%$ incidence of celiac disease [32]. Immunotherapy induced celiac disease was proposed to be defined as grade 2 toxicity, but questions remain whether the disease is only triggered through ICI treatment or is genuinely induced by immunotherapy; the recommendation of the authors would be to suspect ICI induced celiac disease if symptoms start shortly after cancer therapy initiation. Fortunately, treatment in the cases reported was represented by a gluten free diet, with no need for corticosteroids or discontinuation of immunotherapy [80].

Alongside mild side effects that usually respond to therapy and have no significant impact on the outcomes of these patients, there are, unfortunately, rare cases with severe and fatal events. Prompt diagnosis and therapy may aid in improving consequences for this particular category of patients. A life-threatening complication probably due to tumoral flare from nivolumab therapy was described in a patient with metastatic pulmonary adenocarcinoma. A routine CT scan revealed gastric perforation; due to lack of symptomatology, conservatory treatment was chosen, together with discontinuation of immunotherapy [30]. Another similar case of occult gastric perforation in a patient presenting with abdominal pain was reported; the patient was under bevacizumab, carboplatin, paclitaxel therapy, along with an
PD-L1 inhibitor, namely, atezolizumab as third line treatment for non-small-cell lung cancer. The authors suggested that the gastrointestinal perforation was due to the synergistic toxicity of the medications taken, a causal role for only one of them being difficult to establish. The toxicity was fatal for the patient, he died 4 weeks after diagnosis with massive hematemesis. Although uncommon, hematemesis can be a sign of gastric perforation; the authors pointed out that even though immunotherapy was stopped, the perforation did not heal, leading up to a massive hemorrhage finally, although digestive hemorrhage is not commonly accompanying the perforation [31].

Lower gastrointestinal symptoms which are frequently reported in ICI treatment consist of diarrhea, constipation, lower abdominal pain. Diarrhea is classified in four severity grades from mild to very severe. Very severe forms may evolve with perforations and hemorrhages. At colonoscopy with biopsy, main findings are represented by intraepithelial lymphocytes (CTLA-4 inhibitors), granulomas, atrophy and even rupture of crypts (anti-PD1). Severe forms necessitate fluids and electrolytes replacement and corticosteroids treatment. In cases not responding to corticosteroids, infliximab, mycophenolate mofetil or salicylates can be useful. Microbiota transplants were reported as possibly being helpful in severe cases, although there is scarce data regarding this procedure for immune-related effects, and only two cases reported, both successful in reducing the symptomatology; more studies are required in order to make a proper recommendation $[79,84]$.

Extensive reviews have shown that ICI treatment often leads to colitis [85]. The ICI related colitis must be differentiated from colitis of other etiologies: infections, inflammatory intestinal diseases, graft versus host disease, drug induced or IBD. The latter one may present similar findings at colonoscopy. Colitis may be asymptomatic, but it can also clinically manifest with mild symptoms (mild pain, pathological products in stool), with severe symptoms (intense pain, peritonitis), or life threatening [78].

Apart from the findings described above concerning the lower gastrointestinal symptoms, rare events involving the bowel have also been reported.

A 47-year-old patient with pulmonary adenocarcinoma treated with nivolumab and ipilimumab developed severe enterocolitis, refractory to first line treatment with corticosteroids. Although colitis is one of the most common immune-related adverse effects reported, severe cases are not common. Eventually he was successfully treated with infliximab, with good response [34]. Another case reported of a metastatic melanoma, treated with the same combination therapy, also developed corticosteroids refractory colitis that only improved after infliximab treatment [35]. One case of an infliximab refractory colitis was also described in a patient treated with nivolumab; in follow up, the patient developed bowel perforation that required total colectomy with terminal ileostomy; the patient eventually died from sepsis 35 days after the colectomy was performed [36]. When speaking about ICI induced colitis, although the usual histologic and endoscopic findings resemble that of the IBD, there are also reports of some unusual cases such as collagenous colitis after pembrolizumab and atezolizumab therapy $[37,38]$. 
A case of pseudomembranous colitis in a 57-year-old patient with hepatocellular carcinoma was reported, contributing to the constellation of different manifestations and endoscopic findings that can occur with anti PD-1 therapy [39]. These cases also highlight the importance of endoscopy in these patients. A case of microscopic colitis induced by pembrolizumab proves that ICI treatment might be included among other medications associated with this disease and that this type of colitis should be considered in the differential diagnosis of a patient presenting with diarrhea that is under immunotherapy [41].

Inflammatory bowel disease responding to mesalazine treatment was described in a 64-year-old male treated with nivolumab. The diagnosis was made after colonoscopy and histologic examination of the biopsies taken, which were consistent with early stage IBDs. The late onset of the disease, 15 months after the beginning of the therapy underlines the necessity for exhaustive evaluation of this category of patients, keeping in mind that the adverse effects can also happen during or even after cessation of therapy [42]. A team of researchers pointed out the existence of other 9 cases of IBD after combined treatment with nivolumab and metformin, pointing out the need for further investigations [43]. The authors point out that while little is known about the real adverse effects that are caused by the combined treatment, a higher incidence of IBD was detected in patients with diabetes mellitus and lung cancer receiving this type of treatment. Given the fact that IBD can significantly influence a patient's quality of life, further studies are needed to establish the causal role in the appearance of the disease for both types of medication. Tremelimumab was also found to induce IBD in different cancer treated patients [44]. Another team of researchers described a case of granulomatous enterocolitis due to combined ipilimumab and nivolumab treatment, which interestingly did not present with diarrhea, but with hematochezia and fever, which eventually required hemicolectomy [40].

A single case reported until now described the development of eosinophilic enteritis in a melanoma patient treated with dual therapy (nivolumab+ipilimumab) [51]. Protein losing enteropathy related to anti PD-1 therapy was described in one case report; it led to ceasing the ICI treatment and only providing supporting care [52]. Another case of widespread enteropathy with nivolumab treatment was reported, leading to drug discontinuation [53]. In this case, a lung cancer patient presented with diarrhea, after more than 2 years of nivolumab treatment. Colonic biopsies throughout the colon, including the ileum, revealed the presence of collagenous colitis. Nivolumab was stopped until symptomatology resolution, and then started again. Shortly after, the patient developed nausea and epigastric pain, and after thorough investigations, the medical team concluded that the histologic probes were consistent with iatrogenic duodenitis and colitis. The authors pointed out that this is the first case reported of a widespread immune infiltration in almost the entire bowel. Nivolumab treatment was discontinued with complete symptom resolution and no effect on the patient's prognosis at a two-year followup. Apoptotic enteropathy due to pembrolizumab was additionally described [54]. The 62-year-old woman following ICI treatment for lung cancer complained about epigastric pain, nausea, diarrhea, 9 months after pembrolizumab was started. Biopsies taken from the duodenum as well as the colon were consistent with apoptotic induced enteropathy, given the fact that epithelial apoptosis was predominant in all the probes examined. Patient's symptoms improved after drug discontinuation. Other cases of enteritis were also reported [55]. Physicians must be aware of the wide spectrum of endoscopic and pathologic findings in this category of patients, in order to properly diagnose and treat them.

A 40-year-old melanoma patient, following treatment with nivolumab and ipilimumab has undergone surgery for silent appendicitis; the diagnosis was made after a routine PET scan, correlated with the biological evaluation. The case revealed the large spectrum of immune-related adverse effects that can even be missed or diagnosed by chance [45]. Another interesting side effect involving the small bowel was reported in a 56-year-old patient with metastatic melanoma, under treatment with nivolumab and ipilimumab, who developed small bowel perforation due to fast tumor regression after immunotherapy, revealing the multitude of possibilities when it comes to ICI related adverse effects [46]. We found other four cases of nivolumab, ipilimumab or atezolizumab treated patients that developed small bowel perforation due to ICI treatment that were also reported in the literature [47-50]. A 62 -year-old male receiving third line treatment with nivolumab for lung adenocarcinoma developed intestinal pseudoobstruction; he responded to intravenous corticosteroids; the case adds to the diversity of immune related side effects that can appear [56]. An interesting case-report on a complication related to ICI use revealed that physicians should be aware not only of acute immune-related adverse events, but also of the chronic ones. A 73-year-old female with metastatic gastroesophageal junction adenocarcinoma developed chronic intestinal pseudo-obstruction, following treatment with pembrolizumab. Paraneoplastic syndrome, inflammation or infection were excluded. Her symptomatology persisted even after discontinuation of treatment, leading to another two presentations in the emergency unit for postprandial nausea and vomiting, so a decision to stop cancer treatment until resolution was taken [57]. There are only two cases in literature mentioning this type of complication after ICI.

Regarding more severe cases related to the lower gastrointestinal tract, a fatal case of myenteric plexus neuropathy that led to gastrointestinal hypomotility, intestinal perforation and eventually sepsis was described in a 66-yearold female after a single dose of ipilimumab and nivolumab for Merkle cell carcinoma [58]. The authors suggest that late initiation of corticosteroids (day 22 after first symptom occurred) may have contributed to the fatal consequence. Intestinal paresis after ICI therapy was reported by the same team of authors in another case: a patient who developed severe constipation caused by myenteric ganglionitis after ipilimumab, suggesting that cases of hypomotility need to be closely investigated, since they can lead to potentially irreversible outcomes [59].

The prevalence of hepatic toxicity varies between 6.4 and $7.1 \%$ depending on the type of ICI, being more frequent in anti CTLA- 4 treatment. When ICIs are used in combination, toxicity can increase up to $30 \%$, the most severe forms being reported in up to $18.8 \%$ of patients $[86,87]$. For instance, 
nivolumab may be hepatotoxic in up to $10 \%$ of cases, but if associated with ipilimumab, up to one third of patients may develop hepatotoxicity [88]. In general, hepatotoxicity occurs one to three months after beginning the ICI therapy.

Hepatitis secondary to ICI treatment may be asymptomatic or symptomatic, but most of the times it lacks symptoms, the diagnosis being set on the increased level of serum aminotransferases, or a cholestatic syndrome. If symptomatic, fever, anorexia, jaundice, nausea, vomiting, abdominal pain, hemorrhages are present. Differential diagnosis with other liver diseases (secondary cancers, viral, alcohol or drug induced liver disease) can be quite difficult; imaging methods are helpful for the exclusion of liver metastasis or, in patients with cholestasis, for the assessment of bile ducts and exclusion of an obstructive jaundice due to stones or tumors $[89,90]$. In this situation, abdominal ultrasound or even MRCP can help the diagnosis. Otherwise, in the majority of patients with mild forms, no modifications will be noticed at the ultrasound. In severe forms, the ultrasound can document liver enlargement, high echogenicity with parenchymal attenuation, periportal edema and reaction of local lymph nodes. The same modifications can be described by a CT scan, but with a higher accuracy [90].

At present, a five-step grading system is in use for defining the severity of liver toxicity, a system very useful in treating these patients. Grade 1 includes asymptomatic patients with a slight increase of aminotransferases and bilirubin. This mild form of hepatitis does not require any treatment, and chemotherapy should not be interrupted. Grade 2 includes patients with a 3-5 folds increase in aminotransferases. It may need corticosteroids and temporary interruption of chemotherapy. Grade 3 hepatitis is symptomatic, with marked elevation of aminotransferases ( 5 to 20 folds). Grade 4 is also symptomatic, with elevations of more than 20 folds in serum aminotransferases. In these cases, the National Cancer Network management guide recommends discontinuing the ICI therapy and giving immunosuppressive therapy (cortisone, mycophenolate mofetil, anti-thymocyte globulin, tacrolimus [78, 91]. In severe cases, plasma exchange may also by lifesaving.

Regarding rare hepatic side effects of checkpoint inhibitor therapy, nivolumab induced cholangitis is one of them. A fatal case of sclerosing cholangitis was reported in a 77-year-old male who did not respond to corticosteroids; the authors suggested that ICI treatment led to resistance to immunosuppressive treatment besides the life-threating side effect [60].

A single case of nonalcoholic fatty liver disease (NAFLD) and lipodystrophy was reported in a 45-year-old melanoma patient receiving nivolumab. Although the exact causal role was difficult to establish, the development of lipodystrophy and then the appearance of NAFLD, with exclusion of other causes for liver damage, led the authors to believe that this could be a new side-effect of anti PD-1 therapy [61].

Pembrolizumab induced liver injury with predominantly biliary damage was also recounted, with good response to corticosteroids. The authors point out the importance of liver biopsy in some cases, since for this patient, the damage was not clear after biological and imaging evaluation: investigators excluded all possible causes for liver injury, then proceeded to computer tomography and MRCP which only showed diffuse hepatomegaly and endoscopic retrograde cholangiopancreatography that excluded sclerosing cholangitis [62]. To this effect, a clinicopathological study on 10 patients treated with pembrolizumab or atezolizumab revealed the existence of granulomatous hepatitis, sclerosing cholangitis or lymphocytic duct injury; therefore, a large spectrum of liver injury is associated with ICI therapy, besides hepatitis [63]. The patients presented with markers of liver injury in a median of 3.5 weeks after treatment with pembrolizumab or atezolizumab was started, and only two patients presented with high levels of IgG. All patients presented with elevated hepatobiliary enzymes, although 4 were asymptomatic. Also, of all 10 patients, antinuclear antibodies $(1 / 80)$ were identified in two of them. Two patients presented with abnormal findings on liver imaging, namely diffuse cholangiopathy. There are no clear indications for performing liver biopsy in patients with suspected or confirmed ICI induced liver injury and histology results are not always in harmony with clinical presentation. Each case should be treated by a team of experts, and the decision for a liver biopsy should be made if it benefits the patient. A particularly rare side effect has also been reported in the last year: two cases of hepatic sinusoidal obstruction syndrome related to nivolumab treatment. A 66-yearold female and a 65-year-old male, both with melanoma undergoing nivolumab therapy, and both cases presenting portal hypertension and ascites. A complete biochemical and imaging work-up was performed, before carrying out the liver biopsy, which eventually confirmed the diagnosis. The venoocclusive disease, in these cases, could be explained by the infiltration of T-cells in the hepatocytes, causing obstruction of the terminal hepatic venules and leading to symptoms of portal hypertension. Although it is a rare cause of ascites, in general, these cases highlight the need for a complete panel of investigations for patients undertaking ICI treatment $[64,65]$.

A patient with metastatic renal cell carcinoma was treated with pembrolizumab, but due to progression of the disease, he received pazopanib, a tyrosine-kinase inhibitor. He developed a vanishing bile duct syndrome which proved to be fatal. The authors raised the question whether the previous treatment with pembrolizumab might have contributed substantially to the hepatotoxicity, especially considering its long half-life [66].

Pancreatitis due to ICI treatment is a rare event $[92,93]$. Its onset varies between 2 and 12 weeks from the start of the therapy and a definite treatment is not available yet. A report on a patient following nivolumab treatment for non-small lung cancer found no changes of the pancreas in imagery methods in the acute phase. The diagnosis of acute pancreatitis was possible because of specific symptomatology and elevated amylase and lipase serum levels. In day 41 after diagnosis, a follow up MRCP was performed, revealing slight pancreatic swelling. The authors suggested the theory that immune-related pancreatitis could be a subacute event and imaging could be inconclusive at presentation [68].

It has been documented that PD-1 and PD-L1 inhibitors precipitate diabetes in animal models [94] and a review on patients under PD-1 or PD-L1 inhibitors found a $0.9 \%$ prevalence of treatment induced insulin-dependent diabetes [67]. Marchand et. al [69] published an article revealing the case of a 55-year-old patient treated with nivolumab who developed severe pancreatic atrophy and fulminant diabetes 
mellitus, then proceeding to analyze 6 more cases of PD-1 inhibitor induces diabetes, in order to better understand the pathophysiology involved in this particular side effect [70]. Pancreas volume was decreased from the start in 2 cases of fulminant diabetes, and during follow-up, a gradual decrease in volume was likewise observed in the other four cases of ICI induced diabetes [71]. The basic mechanism behind this effect is based on the disruption of inhibitory signals to T cells and consequent intrusion of T cells within organs; this leads to an immune-activated response that entirely abolishes beta cells [72]. Byun et al. [73] retrospectively reviewed 18 patients with PD-1/PD-L1 induced diabetes mellitus and proposed common characteristics for this group: the onset of diabetes is abrupt, the process appears to be irreversible, all patients develop pancreas atrophy and those who have GAD65 positive antibodies appear to develop diabetes early during treatment. Sometimes, pancreas atrophy is accompanied by thyroid atrophy and hypothyroidism and other immune adverse effects such as immobilizing polyarthritis as was described in a 72-year-old man with metastatic melanoma [74].

Rawson et al. [75] report the unusual case of a pembrolizumab induced pancreatitis presenting as small bowel obstruction; the diagnosis was made after surgery and histopathological examination, which revealed significant damage to the pancreas. The authors point out that the risks associated with immunotherapy can be higher than realized, since some of them are asymptomatic and remain undetected [75].

A case of diarrhea in a pembrolizumab treated patient proved that not all cases are caused by colitis; in this case, it was exocrine pancreatic insufficiency, developed after an episode of autoimmune pancreatitis [76]. The case illustrates the necessity of complete evaluation of these patients since pancreatitis was not accompanied by specific symptoms [77].

\section{CONCLUSIONS}

The mechanism by which immunotherapy provides clinical efficacy, by amplifying T-cell response against the tumor cells leads to an imbalance of the immunological responses of the body, subsequently precipitating the appearance of various immune-related events. Gastro-intestinal toxicity related to ICI treatments is increasing and can even be fatal, in some cases. A keen insight into the pathologic mechanisms that can lead to frequent and rare side effects, as well as knowledge of the possible signs and symptoms are needed, in order to accurately develop treatment guidelines. Certain side effects have a low incidence, which makes it difficult to associate them with checkpoint inhibitor therapy, but they represent major clinical issues for both medical staff and patients. Each of the severe reactions mentioned above needed to be abundantly evaluated before placing the right diagnosis, creating a burden on the healthcare system, as well as diminishing life expectancy for patients. This emphasizes the need for continuous research in the field of common and rare side effects related ICI.

Conflicts of interest: None to declare.

Authors' contributions: L.R., D.C. and D.R conceived and designed the study, analyzed the data and wrote the draft. C.G. collected the data and contributed to the writing of the manuscript. D.C. and D.R. revised the manuscript. All authors critically revised the manuscript, approved the final version to be published, and agree to be accountable for all aspects of the work.

Supplementary material: To access the supplementary material visit the online version of the $J$ Gastrointestin Liver Dis at http://dx.doi. org/10.15403/jgld-3671.

\section{REFERENCES}

1. Busch W. Aus der Sitzung der medicinischen Section vom 13 November 1867. Berlin Klin Wochenschr 1868;5:137.

2. Cancer Research Institute. FDA Approves New Immunotherapy for Metastatic Melanoma. New York. [Cited 2021 March 21]. Available from: https://www.cancerresearch.org/en-us/news/2011/fda-approvesnew-immunotherapy-for-melanoma

3. Adachi K, Tamada K. Immune checkpoint blockade opens an avenue of cancer immunotherapy with a potent clinical efficacy. Cancer Sci 2015:106:945-950. doi:10.1111/cas.12695

4. Ribas A, Wolchok JD. Cancer immunotherapy using checkpoint blockade. Science 2018:359:1350-1355. doi:10.1126/science.aar4060

5. Samaan MA, Pavlidis P, Papa S, Powell N, Irving PM. Gastrointestinal toxicity of immune checkpoint inhibitors: from mechanisms to management. Nature Rev Gastroenterol Hepatol 2018;15:222-234. doi:10.1038/nrgastro.2018.14

6. Hodi FS, O'Day SJ, McDermott DF, et al. Improved survival with ipilimumab in patients with metastatic melanoma. N Engl J Med 2010;363:711-723. doi:10.1056/NEJMoa1003466

7. US National Library of Medicine. ClinicalTrials.gov. Phase II Study for Previously Untreated Subjects With Non Small Cell Lung Cancer (NSCLC) or Small Cell Lung Cancer (SCLC). 2012. Available from: https://clinicaltrials.gov/ct2/show/NCT00527735

8. US National Library of Medicine. ClinicalTrials.gov. First-Line Gemcitabine, Cisplatin + Ipilimumab for Metastatic Urothelial Carcinoma. 2017. Available from: https://clinicaltrials.gov/ct2/show/ NCT01524991

9. US National Library of Medicine. ClinicalTrials.gov. Study of MDX010 in Patients With Metastatic Hormone-Refractory Prostate Cancer. 2014. Available from: https://clinicaltrials.gov/ct2/show/ NCT00323882

10. Kim, BJ, Jang HJ, Kim HS, Kim JH. Current Status of Immune Checkpoint Inhibitors in Gastrointestinal Cancers. J Cancer 2017;8:1460-1465. doi:10.7150/jca.18470

11. Vaddepally RK, Kharel P, Pandey R, Garje R, Chandra AB. Review of Indications of FDA-Approved Immune Checkpoint Inhibitors per NCCN Guidelines with the Level of Evidence. Cancers (Basel) 2020;12:738. doi: $10.3390 /$ cancers 12030738

12. Khoja L, Day D, Wei-Wu Chen T, Siu LL, Hansen AR. Tumour- and class-specific patterns of immune-related adverse events of immune checkpoint inhibitors: a systematic review. Ann Oncol 2017;28:23772385. doi:10.1093/annonc/mdx286

13. Cabrita R, Lauss M, Sanna A, et al. Tertiary lymphoid structures improve immunotherapy and survival in melanoma. Nature 2020;577:561-565. doi:10.1038/s41586-019-1914-8

14. Postow MA, Sidlow R, Hellmann MD. Immune-related adverse events associated with immune checkpoint blockade. N Engl J Med 2018;378:158-168. doi:10.1056/NEJMra1703481 
15. Gupta A, De Felice KM, Loftus EV Jr, Khanna S. Systematic review: colitis associated with anti-CTLA-4 therapy. Aliment Pharmacol Ther 2015;42:406-417. doi:10.1111/apt.13281

16. Larkin J, Chiarion-Sileni V, Gonzalez R, et al. Combined nivolumab and ipilimumab or monotherapy in untreated melanoma. N Engl J Med 2015;373:23-34. doi:10.1056/NEJMoa1504030

17. Marin-Acevedo JA, Chirila RM, Dronca RS. Immune Checkpoint Inhibitor Toxicities. Mayo Clin Proc 2019;94:1321-1329. doi:10.1016/j. mayocp.2019.03.012

18. Ellithi M, Elnair R, Chang GV, Abdallah MA. Toxicities of Immune Checkpoint Inhibitors: Itis-Ending Adverse Reactions and More. Cureus 2020;12:e6935. doi:10.7759/cureus.6935

19. Endo R, Nakamura Y, Ishizuki S, et al. Ulcerative esophagitis associated with combined nivolumab and ipilimumab therapy. J Dermatol 2020;47:e299-e300. doi:10.1111/1346-8138.15421

20. tsumi T, Tsubouchi K, Harada T. Refractory Severe Esophagitis During Durvalumab Therapy in a Patient With Locally Advanced NSCLC. J Thorac Oncol 2019;14:e40-e42. doi:10.1016/j.jtho.2018.10.153

21. Horisberger A, La Rosa S, Zurcher JP, et al. A severe case of refractory esophageal stenosis induced by nivolumab and responding to tocilizumab therapy. J Immunother Cancer 2018;6:156. doi:10.1186/ s40425-018-0481-0

22. Zhu AX, Finn RS, Edeline J, et al. Pembrolizumab in patients with advanced hepatocellular carcinoma previously treated with sorafenib (KEYNOTE-224): a non-randomised, open-label phase 2 trial. Lancet Oncol 2018;19:940-952. doi:10.1016/S1470-2045(18)30351-6

23. Acero Brand FZ, Suter N, Adam JP, et al. Severe immune mucositis and esophagitis in metastatic squamous carcinoma of the larynx associated with pembrolizumab. J Immunother Cancer 2018;6:22. doi:10.1186/ s40425-018-0332-Z

24. Vindum HH, Agnholt JS, Nielsen AWM, Nielsen MB, Schmidt H. Severe steroid refractory gastritis induced by Nivolumab: A case report. World J Gastroenterol 2020;26:1971-1978. doi:10.3748/wjg.v26.i16.1971

25. Rovedatti L, Lenti MV, Vanoli A, Feltri M, De Grazia F, Di Sabatino A. Nivolumab-associated active neutrophilic gastritis. J Clin Pathol 2020;73:605-606. doi:10.1136/jclinpath-2020-206540

26. Vandepapelière J, Siplet J, Libbrecht L, Dano H, Baurain JF, Moreels T. Auto-immune gastritis induced by pembrolizumab, an anti-PD-1, in a melanoma patient. Acta Gastroenterol Belg 2020;83:482-484.

27. Yip RHL, Lee LH, Schaeffer DF, Horst BA, Yang HM. Lymphocytic gastritis induced by pembrolizumab in a patient with metastatic melanoma. Melanoma Res 2018;28:645-647. doi:10.1097/ CMR.0000000000000502

28. Kim H, Ha SY, Kim J, Kang M, Lee J. Severe cytomegalovirus gastritis after pembrolizumab in a patient with melanoma. Curr Oncol 2020;27:436-39. doi:10.3747/co.27.6163

29. Kobayashi M, Yamaguchi O, Nagata K, Nonaka K, Ryozawa S. Acute hemorrhagic gastritis after nivolumab treatment. Gastrointest Endosc 2017;86:915-916. doi:10.1016/j.gie.2017.04.033

30. Malipatil B, Palleti A, Verma NS, Katti SV. Gastric perforation due to nivolumab related tumor flare. Indian J Cancer 2019;56:374-375. doi:10.4103/ijc.IJC_776_18

31. Gunjur A, Chong G, Lim A, et al. Occult Gastrointestinal Perforation in a Patient With EGFR-Mutant Non-Small-Cell Lung Cancer Receiving Combination Chemotherapy With Atezolizumab and Bevacizumab: Brief Report. Clin Lung Cancer 2020;21:e57-e60. doi:10.1016/j.cllc.2019.11.014

32. Schoenfeld SR, Aronow ME, Leaf RK, Dougan M, Reynolds KL. Diagnosis and Management of Rare Immune-Related Adverse Events. Oncologist 2020;25:6-14. doi:10.1634/theoncologist.2019-0083
33. Calugareanu A, Rompteaux P, Bohelay G, et al. Late onset of nivolumabinduced severe gastroduodenitis and cholangitis in a patient with stage IV melanoma. Immunotherapy 2019;11:1005-1013. doi:10.2217/imt2019-0077

34. Luque Carmona AM, Ontanilla-Clavijo G, Leo Carnerero E. Severe enterocolitis secondary to ipilimumab and nivolumab with an excellent response to a single dose of infliximab. Rev Esp Enferm Dig 2020;112:889-890. doi:10.17235/reed.2020.6886/2020

35. Karanfilian B, Dalal I, Bhurwal A, Patel AV. Immune-Mediated Colitis due to Concurrent Ipilimumab and Nivolumab Therapy Treated With Infliximab. Am J Ther 2020;27:e415-e417. doi:10.1097/ MJT.0000000000001165

36. Callens R, Tamsin A, van Zandweghe L. Nivolumab-Induced Fulminant Immune-Related Colitis Despite Infliximab in a Patient With NSCLC. J Thorac Oncol 2019;14:e49-e50. doi:10.1016/j.jtho.2018.11.014

37. Calvani J, Elia R, Battistella M, et al. An unusual digestive complication under anti-PD-1 (pembrolizumab). Ann Pathol 2020;40:320-323. doi:10.1016/j.annpat.2020.02.001

38. Gallo A, Talerico R, Novello L, et al. Collagenous colitis and atezolizumab therapy: an atypical case. Clin J Gastroenterol 2021;14:165-169. doi:10.1007/s12328-020-01276-4

39. Kidambi TD, Chu P, Lee JK, Lin JL. Immunotherapy-Associated Pseudomembranous Colitis. Am J Gastroenterol 2019;114:1708 doi:10.14309/ajg.0000000000000282

40. Paton DJW, Warburton L, Chung K, Meniawy TM, Kumarasinghe MP. Ipilimumab- and nivolumab-associated enterocolitis with florid necrotising granulomatous inflammation: a novel manifestation of 'immunomodulatory' enterocolitis. Pathology 2018;50:466-469. doi:10.1016/j.pathol.2017.11.094

41. Ahmed M, Francis G. Pembrolizumab-Induced Microscopic Colitis. Am J Gastroenterol. 2018;113:629-630. doi:10.1038/ajg.2018.8

42. Guaitoli G, Baldessari C, Tomasello C, et al. Late Gastrointestinal Toxicity During Nivolumab Therapy in Non-Small Cell Lung Cancer: A Rare Case of Inflammatory Bowel Disease. J Thorac Oncol 2018;13:e152-e153. doi:10.1016/j.jtho.2018.03.022

43. Zhou H, Liu J, Zhang Y, Zhang L. Inflammatory bowel disease associated with the combination treatment of nivolumab and metformin: data from the FDA adverse event reporting system. Cancer Chemother Pharmacol 2019;83:599-601. doi:10.1007/s00280-018-03763-5

44. Marthey L, Mateus C, Mussini C, et al. Cancer Immunotherapy with Anti-CTLA-4 Monoclonal Antibodies Induces an Inflammatory Bowel Disease. J Crohns Colitis 2016;10:395-401. doi:10.1093/eccojcc/jjv227

45. Mangas C, Leoni-Parvex S, Spataro V. Silent acute appendicitis revealed by PET-CT during treatment with ipilimumab and nivolumab: a new immune-related adverse event. Cancer Immunol Immunother 2020;69:2409-2410. doi:10.1007/s00262-020-02724-3

46. Patel KR, Lee LY, Tripathy A, McKean D. Case of small bowel perforation secondary to nivolumab and ipilimumab related tumour regression. BMJ Case Rep 2020;13:e232304. doi:10.1136/bcr-2019-232304

47. Tso DK, Avery LL, Lev MH, Kamalian S. Nivolumab-induced small bowel obstruction and perforation: a rare but life-threatening side effect of immunotherapy. Emerg Radiol 2020;27:107-110. doi:10.1007/ s10140-019-01731-X

48. Yasuda K, Tanaka T, Ishihara S, et al. Intestinal perforation after nivolumab immunotherapy for a malignant melanoma: a case report. Surg Case Rep 2017:3:94. doi:10.1186/s40792-017-0370-7

49. Mohamed AA, Richards CJ, Boyle K, Faust G. Severe inflammatory ileitis resulting in ileal perforation in association with combination immune 
checkpoint blockade for metastatic malignant melanoma. BMJ Case Rep 2018;2018:bcr2018224913. doi:10.1136/bcr-2018-224913

50. Zillioux J, DiLizia M, Schaheen B, Rustin R, Krupski TL. Ileal perforation in the setting of atezolizumab immunotherapy for advanced bladder cancer. Can J Urol 2018;25:9525-9526.

51. Yang J, Lagana SM, Saenger YM, Carvajal RD. Dual checkpoint inhibitor-associated eosinophilic enteritis. J Immunother Cancer 2019;7:310. doi:10.1186/s40425-019-0743-5

52. Lee SY, Kim MH, Jang M, Park Y, Cho BC. A man with recurrent hypovolemic shock on anti-programmed cell death protein 1 treatment: Immune-related protein-losing enteropathy. Eur J Cancer 2018;104:104107. doi:10.1016/j.ejca.2018.09.015

53. Facchinetti F, Gnetti L, Caruana P, et al. Widespread Nivolumab-induced Enteropathy in a Long Responder Non-Small-cell Lung Cancer Patient. Clin Lung Cancer 2018;19:e591-e596. doi:10.1016/j.cllc.2018.04.013

54. Blesl A, Rainer F, Pollheimer MJ. PD1 inhibitor-induced apoptotic enteropathy in the duodenum. Gastrointest Endosc 2019;90:854-855. doi:10.1016/j.gie.2019.06.023

55. Sanders D, Webber D, Chatur N. Enteritis with immune checkpoint inhibitor use. CMAJ 2019;191:E1106. doi:10.1503/cmaj.190244

56. Fragulidis G, Pantiora E, Michalaki V, et al. Immune-related intestinal pseudo-obstruction associated with nivolumab treatment in a lung cancer patient. J Oncol Pharm Pract 2019;25:487-491. doi: $10.1177 / 1078155217738325$

57. Besaw RJ, Smith MP, Zerillo JA, Bullock AJ. Chronic intestinal pseudoobstruction in a patient with metastatic gastro-oesophageal junction cancer receiving treatment with pembrolizumab. BMJ Case Rep 2019;12:e232388. doi:10.1136/bcr-2019-232388

58. Appelbaum J, Wells D, Hiatt JB, et al. Fatal enteric plexus neuropathy after one dose of ipilimumab plus nivolumab: a case report. J Immunother Cancer 2018;6:82. doi:10.1186/s40425-018-0396-9

59. Bhatia S, Huber BR, Upton MP, Thompson JA. Inflammatory enteric neuropathy with severe constipation after ipilimumab treatment for melanoma: a case report. J Immunother 2009;32:203-205. doi:10.1097/ CJI.0b013e318193a206

60. Yoshikawa Y, Imamura M, Yamaoka K, et al. A case with life-threatening secondary sclerosing cholangitis caused by nivolumab. Clin J Gastroenterol 2021;14:283-287. doi:10.1007/s12328-020-01287-1

61. Eigentler T, Lomberg D, Machann J, Stefan N. Lipodystrophic Nonalcoholic Fatty Liver Disease Induced by Immune Checkpoint Blockade. Ann Intern Med 2020;172:836-837. doi:10.7326/L19-0635

62. Kida A, Matsuda K, Matsuda M, Sakai A. Hepatobiliary and Pancreatic: Biliary injury related to checkpoint inhibitor "pembrolizumab". J Gastroenterol Hepatol 2019;34:1478. doi:10.1111/jgh.14677

63. Zen Y, Chen YY, Jeng YM, Tsai HW, Yeh MM. Immune-related adverse reactions in the hepatobiliary system: second-generation check-point inhibitors highlight diverse histological changes. Histopathology 2020;76:470-480. doi:10.1111/his.14000

64. Charvet E, Lheure C, Isnard C, et al. Hepatic sinusoidal obstruction syndrome induced by nivolumab in advanced melanoma: a case report. Ann Oncol 2020;31:661-662. doi:10.1016/j.annonc.2020.02.004

65. Riveiro-Barciela M, González-Sans D, Marmolejo D, Salcedo MT, Muñoz-Couselo E. Hepatic sinusoidal obstruction syndrome associated with nivolumab: an uncommon adverse event related to immune checkpoint inhibitors. J Gastrointestin Liver Dis 2021;30:171-172. doi:10.15403/jgld-3207

66. Zhong YY, McLean L, Buckle A, Siva S, Tran B. Vanishing bile duct syndrome associated with pazopanib after progression on pembrolizumab. Can J Urol 2020;27:10339-10341.
67. Stamatouli AM, Quandt Z, Perdigoto AL, et al. Collateral damage: insulin-dependent diabetes induced with checkpoint inhibitors. Diabetes 2018;67:1471-1480. doi:10.2337/dbi18-0002

68. Ikeuchi K, Okuma Y, Tabata T. Immune-related pancreatitis secondary to nivolumab in a patient with recurrent lung adenocarcinoma: A case report. Lung Cancer 2016;99:148-150. doi:10.1016/j.lungcan.2016.07.001

69. Marchand L, Paulus V, Fabien N, et al. Nivolumab-induced acute diabetes mellitus and hypophysitis in a patient with advanced pulmonary pleomorphic carcinoma with a prolonged tumor response. J Thorac Oncol 2017;12:e182-e184. doi:10.1016/j.jtho.2017.07.021

70. Marchand L, Thivolet A, Saintigny P, Fabien N, Vouillarmet J, Thivolet C. Anti-programmed death 1 (PD-1) antibodies and the pancreas: a diabetic storm ahead? Diabetes Care 2018;41:638-639. doi:10.2337/ dc17-2243

71. Marchand L, Thivolet A, Dalle S, et al. Diabetes mellitus induced by PD-1 and PD-L1 inhibitors: description of pancreatic endocrine and exocrine phenotype. Acta Diabetol 2019;56:441-448. doi:10.1007/ s00592-018-1234-8

72. Yilmaz M. Nivolumab-induced type 1 diabetes mellitus as an immune-related adverse event. J Oncol Pharm Pract 2020;26:236-239. doi: $10.1177 / 1078155219841116$

73. Byun DJ, Braunstein R, Flynn J, et al. Immune Checkpoint InhibitorAssociated Diabetes: A Single-Institution Experience. Diabetes Care 2020;43:3106-3109. doi:10.2337/dc20-0609

74. Haupt F, Prosch H, Ebner L. Thyroid atrophy and pancreatic involution after cancer Immunotherapy. Rofo 2020;192:688-690. doi:10.1055/a-1108-1934

75. Rawson RV, Robbins E, Kapoor R, Scolyer RA, Long GV. Recurrent bowel obstruction: unusual presentation of pembrolizumab-induced pancreatitis in annular pancreas. Eur J Cancer 2017;82:167-170. doi:10.1016/j.ejca.2017.05.042

76. Koldenhof JJ, Suijkerbuijk KPM. Diarrhoea during checkpoint blockade, not always colitis. Eur J Cancer 2017;87:216-218. doi:10.1016/j. ejca.2017.08.024

77. Naidoo J, Page DB, Li BT, et al. Toxicities of the anti-PD-1 and antiPD-L1 immune checkpoint antibodies. Ann Oncol 2015;26:2375-2391. doi:10.1093/annonc/mdv383

78. Rajha E, Chaftari P, Kamal M, Maamari J, Chaftari C, Yeung SJ. Gastrointestinal adverse events associated with immune checkpoint inhibitor therapy. Gastroenterol Rep (Oxf) 2019;8:25-30. doi:10.1093/ gastro/goz065

79. Grover S, Rahma OE, Hashemi N, Lim RM. Gastrointestinal and hepatic toxicities of checkpoint inhibitors: algorithms for management. Amer Soc Clin Oncol Educ Book 2018;38:13-19. doi:10.1200/EDBK_100013

80. Postow MA, Sidlow R, Hellmann MD. Immune-related adverse events associated with immune checkpoint blockade. N Engl J Med 2018;378:158-168. doi:10.1056/NEJMra1703481

81. Freeman-Keller M, Kim Y, Cronin H, Richards A, Gibney G, Weber JS. Nivolumab in resected and Unresectable metastatic melanoma: characteristics of immune-related adverse events and association with outcomes. Clin Cancer Res 2016;22:886-894. doi:10.1158/1078-0432. CCR-15-1136

82. Abdel-Wahab N, Shah M, Suarez-Almazor ME. Adverse events associated with immune checkpoint blockade in patients with cancer: A systematic review of case reports. PLoS One 2016;11:e0160221. doi:10.1371/journal.pone.0160221

83. Gentile NM, D'Souza A, Fujii LL, Wu TT, Murray JA. Association between ipilimumab and celiac disease. Mayo Clin Proc 2013;88:414417. doi:10.1016/j.mayocp.2013.01.015 
84. Assarzadegan N, Montgomery E, Anders RA. Immune checkpoint inhibitor colitis: the flip side of the wonder drugs. Virchows Archiv 2018;472:125-133. doi:10.1007/s00428-017-2267-Z

85. Cramer P, Bresalier RS. Gastrointestinal and hepatic complications of immune checkpoint inhibitors. Curr Gastroenterol Rep 2017;19:3. doi:10.1007/s11894-017-0540-6

86. Hodi FS, Postow MA, Chesney JA, et al. Clinical response, progression-free survival (PFS), and safety in patients (pts) with advanced melanoma (MEL) receiving nivolumab (NIVO) combined with ipilimumab (IPI) vs IPI monotherapy in CheckMate 069 study. Presented at the ASCO Annual Meeting, Chicago, May 29-June 2, 2015. J Clin Oncol 2015;33(15 suppl):9004-9004. doi:10.1200/ jco.2015.33.15_suppl.9004

87. Robert C, Schachter J, Long GV, et al. Pembrolizumab versus ipilimumab in advanced melanoma. N Engl J Med 2015;372:2521- 2532. doi:10.1056/NEJMoa1503093

88. Kim KW, Ramaiya NH, Krajewski KM, et al. Ipilimumab associated hepatitis: imaging and clinicopathologic findings. Investig New Drugs 2013;31:1071-1077. doi:10.1007/s10637-013-9939-6

89. Alessandrino F, Tirumani SH, Krajewski KM, et al. Imaging of hepatic toxicity of systemic therapy in a tertiary cancer centre: chemotherapy, haematopoietic stem cell transplantation, molecular targeted therapies, and immune checkpoint inhibitors. Clin Radiol 2017;72:521-533. doi:10.1016/j.crad.2017.04.003

90. Suzman DL, Pelosof L, Rosenberg A, Avigan MI. Hepatotoxicity of immune checkpoint inhibitors: An evolving picture of risk associated with a vital class of immunotherapy agents. Liver Int 2018;38:976-987. doi:10.1111/liv.13746

91. Cancer Therapy Evaluation Program. Common Terminology Criteria for Adverse Events (CTCAE) v5.0. Bethesda (MD), 2017. [Cited 2018 Oct 1]. Available from: https://ctep.cancer.gov/protocoldevelopment/ electronic_applications/docs/CTCAE_v5_Quick_Reference_5x7.pdf

92. Dehghani L, Mikail N, Kramkimel N, et al. Autoimmune pancreatitis after nivolumab anti-programmed death receptor-1 treatment. Eur J Cancer 2018;104:243-246. doi:10.1016/j.ejca.2018.09.014

93. Hofmann L, Forschner A, Loquai C, et al. Cutaneous, gastrointestinal, hepatic, endocrine, and renal side-effects of anti-PD-1 therapy. Eur J Cancer 2016;60:190-209. doi:10.1016/j.ejca.2016.02.025

94. Wang J, Yoshida T, Nakaki F, Hiai H, Okazaki T, Honjo T. Establishment of NOD-Pdcd1-/- mice as an efficient animal model of type I diabetes. Proc Natl Acad Sci U S A 2005;102:11823-11828. doi:10.1073/ pnas.0505497102 